\title{
"Atlas" of numerical solutions for star-disk magnetospheric interaction
}

\author{
M. Čemeljić \\ Nicolaus Copernicus Astronomical Center, Bartycka 18, 00-716 Warsaw, Poland \\ e-mail:miki@camk.edu.pl \\ Received 5 November 2018 / Accepted 7 February 2019

\section{ABSTRACT}

\begin{abstract}
Aims. I report results in numerical simulations of star-disk magnetospheric interaction. A thin accretion disk with a corona above a rotating stellar surface is simulated, and a parameter study is performed to find trends in the angular momentum flux. The results are presented for young stellar objects, but they can be rescaled to other objects with similar geometry.

Methods. I performed resistive and viscous magnetohydrodynamic simulations that reached a quasi-stationary state for cases with different parameters. I computed angular momentum fluxes in the different components of the flow to compare the results.

Results. I present the simulation results with the matter density distribution and a sample of the magnetic field lines, gathered in an "Atlas" of solutions. The torque exerted on the star is computed, together with the angular momentum flux that is expelled from the system in the cases with a conical outflow. I find trends in the components of the flow in the part of parameter space with a slowly rotating star.
\end{abstract}

Key words. stars: formation - stars: magnetic field - stars: pre-main sequence

\section{Introduction}

An analytical solution for a viscous accretion disk was given in Shakura \& Sunyaev (1973) a few years after the first numerical solution by Prendergast \& Burbidge (1968). As in many numerical and analytical models that followed, the disk structure was derived separately in radial and vertical direction. The radial structure was computed by equations averaged over the disk thickness and the vertical structure from the hydrostatic equilibrium in the vertical direction. In some models, radiative transfer was also taken into account.

Height-averaged computations were shown to be insufficient in the analytical work by Urpin (1984) and in many numerical works as well, starting with Kley \& Lin (1992). In all of them, a backflow in the disk midplane occurs that is thought to be of thermal origin.

An analytical solution for a thin accretion disk in three dimensions was given in Kluzniak \& Kita (2000; hereafter KK00). They derived the equations of a polytropic, viscous hydrodynamical accretion disk using the Taylor expansion in the small parameter $\epsilon=H / R$, the disk aspect ratio. Although thermal effects were neglected, backflow is still present with the values of the viscosity parameter $\alpha_{\mathrm{v}}<0.685$. Such a disk accretes only at the higher latitudes, closer to the surface, and the backflow in the mid-plane occurs at a particular distance from the star. Inside this distance, matter always flows toward the star. If $\alpha_{\mathrm{v}}>0.685$, the disk accretes across the entire height.

When stellar magnetic field and disk resistivity are added in the model, the angular momentum transfer depends on the details of star-disk magnetospheric interaction. As first shown in Ghosh \& Lamb (1979a,b), to correctly describe this interaction, it is not enough to consider the disk alone. The rotating stellar surface and corona have to be included in the model so that the interaction with the region in the disk below and beyond the corotation radius is included. Pioneering such simulations were Romanova et al. (2009, 2013) and Zanni \& Ferreira (2009, 2013). As described in Zanni \& Ferreira (2009; hereafter ZF09), to answer the stellar angular momentum problem, the different regimes of magnetospheric interaction need to be probed through exploration of the parameter space. Here I follow this suggestion.

In Sect. 2 I demonstrate the difference in the matter density distribution in the results with different parameters, obtained from a set of simulations sweeping through the parameter space. In Sect. 3 the results with different parameters in the simulations are compared and trends are illustrated, with conclusions listed in Sect. 4. A short but detailed technical exposition of the code setup is presented in the appendix.

\section{Simulations of star-disk magnetospheric interaction}

Using the PLuTo code (v.4.1; Mignone et al. 2007, 2012), I performed simulations of star-disk magnetospheric interaction (SDMI). Initial conditions in the disk and corona and boundary conditions at the edges of the computational domain closely follow ZF09. See the appendix for the details of the numerical setup.

Simulations span over 64 points in the parameter space (see Table 1 ), by varying stellar angular velocity $\Omega_{\star}$, expressed in units of stellar breakup angular velocity $\Omega_{\mathrm{br}}=\sqrt{G M_{\star} / R_{\star}^{3}}$, stellar magnetic field strength $B_{\star}$, and the magnetic Prandtl number

$P_{\mathrm{m}}=\frac{2}{3} \frac{\alpha_{\mathrm{v}}}{\alpha_{\mathrm{m}}}$

where $\alpha_{\mathrm{m}}$ is the resistive parameter. In the simulations presented here, the viscosity parameter was fixed to $\alpha_{\mathrm{v}}=1$.

To compare the results in the different points in the parameter space, a quasi-stationary state in each of the simulations was found by computing the mass flux $\dot{M}$ and the angular momentum 

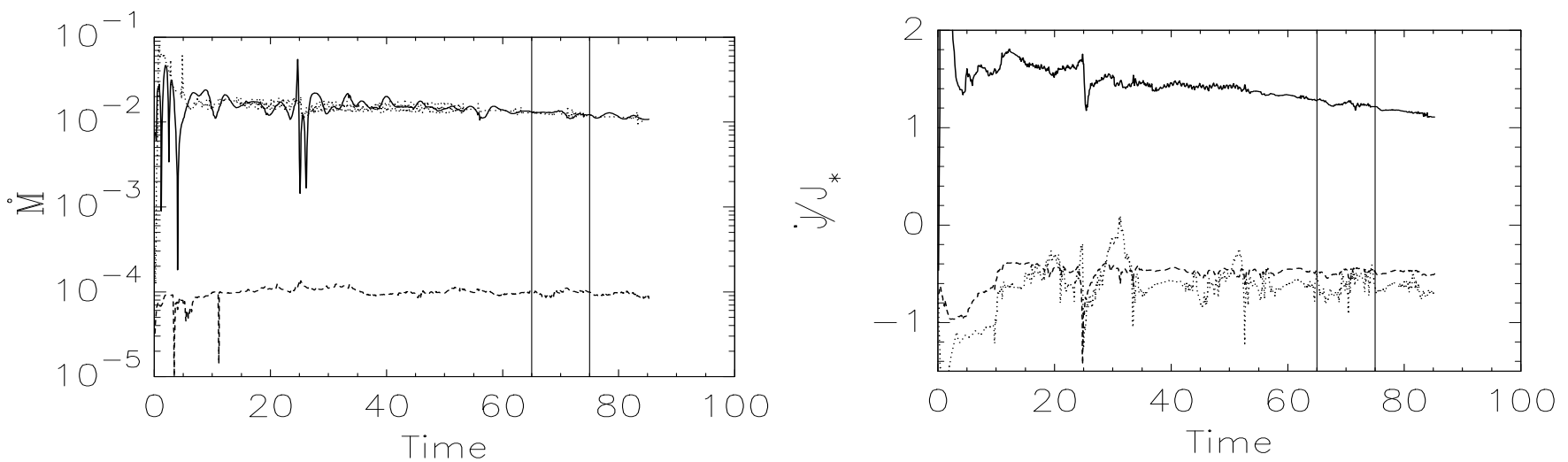

Fig. 1. Fluxes for the case with $\Omega_{\star}=0.1 \Omega_{\mathrm{br}}, B_{\star}=0.5 \mathrm{kG}$, and $\alpha_{\mathrm{m}}=1$. Left panel: mass flux across the disk at $R=12 R_{\star}$ (solid line), onto the star (dotted line) and into the stellar wind (dashed line) in units of $\dot{M}_{0}=\rho_{\mathrm{d} 0} R_{\star}^{2} v_{\mathrm{K} \star}$. Right panel: angular momentum flux of the matter in-falling onto the star from the distances below (solid line) and beyond (dashed line) the corotation radius and in the stellar wind (dotted line) in units of stellar angular momentum. Vertical solid lines mark the interval in time in which an average is taken to compute the quasi-stationary state.

Table 1. Parameter space in the study presented in the "Atlas" for YSOs.

\begin{tabular}{lccccc}
\hline \hline$\Omega_{\star} / \Omega_{\mathrm{br}}$ & $B_{\star}(\mathrm{G})$ & $P_{\mathrm{m}}$ & $\alpha_{\mathrm{m}}$ & $P_{\star}($ days $)$ & $R_{\text {cor }}\left(R_{\star}\right)$ \\
\hline 0.05 & 250 & 6.7 & 0.1 & 9.2 & 7.37 \\
0.1 & 500 & 1.67 & 0.4 & 4.6 & 4.64 \\
0.15 & 750 & 0.95 & 0.7 & 3.1 & 3.54 \\
0.2 & 1000 & 0.67 & 1.0 & 2.3 & 2.92 \\
\hline
\end{tabular}

Notes. The table lists: stellar angular velocity $\Omega_{\star}$, stellar dipole magnetic field strength $B_{\star}$, and the magnetic Prandtl number $P_{\mathrm{m}}$, for which the corresponding resistivity parameter $\alpha_{\mathrm{m}}$ is listed as well, followed by the stellar rotation period and corotation radius.

flux $\dot{J}$. The fluxes were computed by integrating

$$
\dot{M}=\int_{\mathrm{S}} \rho \boldsymbol{v}_{\mathrm{p}} \cdot \mathrm{d} \boldsymbol{S}, \dot{J}=\int_{\mathrm{S}}\left(r \rho v_{\varphi} \boldsymbol{v}_{\mathrm{p}}-\frac{r B_{\varphi} \boldsymbol{B}_{\mathrm{p}}}{4 \pi}\right) \cdot \mathrm{d} \boldsymbol{S},
$$

over the corresponding surface $S$ in the different parts of the flow.

I computed the mass and angular momentum fluxes onto the star, into the stellar wind, and across the disk height in the middle part of the disk at $R_{\mathrm{d}}=12 R_{\star}$. When an outflow formed, the fluxes that contributed to it were also computed at this distance. In the angular momentum flux onto the star, the parts coming from beyond and below the corotation radius $R_{\text {cor }}=\left(G M_{\star} / \Omega_{\star}^{2}\right)^{1 / 3}$ were computed separately. The sign convention is such that a positive angular momentum flux increases the total in the system and a negative decreases it. In both fluxes, the equatorial symmetry was taken into account so that they were computed in a complete meridional plane.

A typical example, computed in one point of the parameter space in the simulations, is presented in Fig. 1. It shows that after relaxation from the initial and boundary conditions (lasting typically for 10-50 stellar rotations), the quasi-stationary state is reached. Oscillations in the simulations, still present into the quasi-stationary state, are smoothed out by averaging over some characteristic interval; typically, an average was taken over ten stellar rotations. In the considered example the averaging interval is from 65 to 75 stellar rotations.

Such an interval in which neither mass nor angular momentum fluxes varied strongly was chosen in each of the 64 simulations. I computed the average values of the angular momentum flux in the various components in the flow during that time interval.
The density distribution in each of the 64 cases can be represented by one of the three solutions from Fig. 2:

- Disk connected with the star by an accretion column (DC).

- Disk pushed away from the star, without an accretion column (D).

- Conical outflow above an accretion column connecting star and disk (DCE).

I label the three cases obtained in the quasi-stationary state in the simulations as D, DC and DCE: D is the disk alone, DC is with disk and column, and DCE is with the disk, column, and magnetospheric ejection present in the result. This signature is used to form an "Atlas" of solutions in Table 2. The numerical values for density and exact positions of the field lines in each of the 64 simulations are different, but they qualitatively resemble the geometry of the flow components in one of the three listed cases.

\section{Trends in the angular momentum flux}

After indicating the quasi-stationary states in simulations, I proceed to compare solutions presented in the "Atlas". In the first example, Fig. 3 shows a comparison of the density in the middle part of the disk in the simulations with an increasing stellar magnetic field. The density increases with increasing magnetic field. This trend contributes to the trends in mass and angular momentum fluxes.

In the second example, the simulations are compared with the increasing stellar rotation rate, in which the stellar magnetic field remains unchanged. Angular momentum flux from the accretion disk contributes to various components of the flow: to the star through the accretion column, to the stellar wind, and to the conical outflow when it forms.

By comparing the solutions, I find a trend in the angular momentum flux, as shown in Fig. 4. With the increase in stellar rotation rate, the spin-up of the star by the infalling matter decreases and eventually switches to a spin-down. A similar outcome is obtained in each of the cases with different stellar magnetic field strengths. This trend is expected because for slowly rotating stars the torque exerted on the star depends mostly on the stellar magnetic field at each value of the resistivity parameter. The trend changes only after a change in flow geometry.

The third example of a trend in the results is in the cases with $\alpha_{\mathrm{m}}=0.1$, in which a conical outflow is formed. The angular momentum flux expelled from the system with such an outflow in the cases with different magnetic field strength is shown in Fig. 5. 


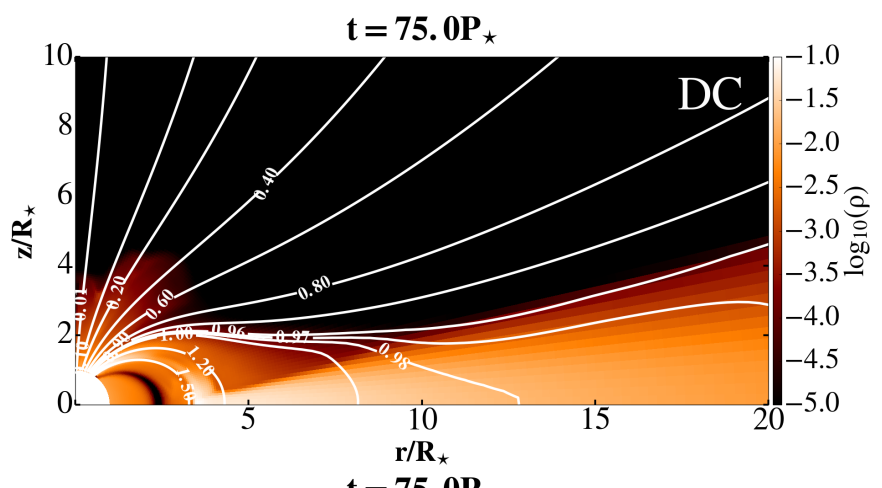

Table 2. Parameter space in the simulations, with an "Atlas" of solutions, where the geometry of the flow components in each case is labeled with D for disk, DC for disk+column, and DCE for disk+column+ejection.

\begin{tabular}{|c|c|c|c|c|}
\hline$\alpha_{\mathrm{m}}=$ & 0.1 & 0.4 & 0.7 & 1 \\
\hline \multicolumn{5}{|c|}{$\Omega_{\star} / \Omega_{\mathrm{br}}$} \\
\hline \multicolumn{5}{|c|}{$B_{\star}=250 \mathrm{G}$} \\
\hline 0.05 & DCE & $\mathrm{DC}$ & DC & DC \\
\hline 0.1 & DCE & DC & DC & DC \\
\hline 0.15 & DCE & DC & DC & DC \\
\hline 0.2 & DCE & $\mathrm{DC}$ & DC & DC \\
\hline \multicolumn{5}{|c|}{$B_{\star}=500 \mathrm{G}$} \\
\hline 0.05 & DCE & $\mathrm{DC}$ & DC & DC \\
\hline 0.1 & DCE & DC & DC & DC \\
\hline 0.15 & DCE & DC & DC & DC \\
\hline 0.2 & DCE & DC & DC & DC \\
\hline \multicolumn{5}{|c|}{$B_{\star}=750 \mathrm{G}$} \\
\hline 0.05 & DCE & $\mathrm{DC}$ & DC & DC \\
\hline 0.1 & DCE & DC & DC & DC \\
\hline 0.15 & DCE & DC & DC & DC \\
\hline 0.2 & DCE & $\mathrm{DC}$ & DC & DC \\
\hline \multicolumn{5}{|c|}{$B_{\star}=1000 \mathrm{G}$} \\
\hline 0.05 & DCE & $\mathrm{DC}$ & DC & DC \\
\hline 0.1 & DCE & DC & DC & DC \\
\hline 0.15 & DCE & $\mathrm{D}$ & $\mathrm{D}$ & $\mathrm{D}$ \\
\hline 0.2 & DCE & $\mathrm{D}$ & $\mathrm{D}$ & $\mathrm{D}$ \\
\hline
\end{tabular}

Notes. In all the cases, $\alpha_{\mathrm{v}}=1$.

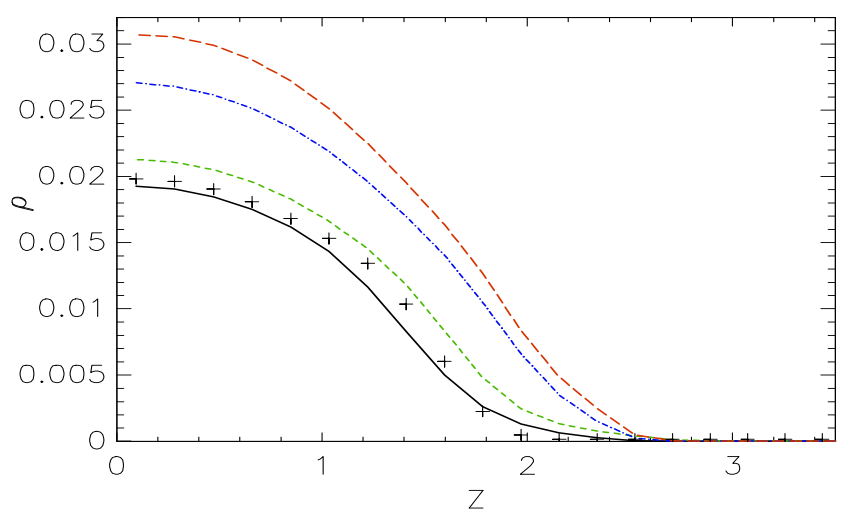

Fig. 3. Disk density in the simulations with $\Omega_{\star}=0.2 \Omega_{\mathrm{br}}$ and $\alpha_{\mathrm{m}}=1$, measured along the disk height at $R=12 R_{\star}$. Results for $B_{\star}=0.25$ (solid black line), 0.5 (short dashed green line), 0.75 (dash-dotted blue line) and $1 \mathrm{kG}$ (long dashed red line) are shown. There is a trend in density with increasing stellar field. The result in the simulations without a magnetic field is shown with pluses.

except for faster stellar rotation together with a strong magnetic field, when the outflow takes away much more, about an order of magnitude, of the angular momentum from the system. The magnetospheric ejection increases with the stronger magnetic field and faster rotation.

The trends in the examples can be compared with the results in other models and simulations when they become available. If they are found to be robust, these trends can be compared with observations and can be incorporated in star formation models.

\section{Conclusions}

In the numerical simulations of star-disk magnetospheric interaction (SDMI), I investigated the angular momentum flux in the system. In a parameter study, stellar rotation rate, magnetic field, and the disk resistivity were varied to obtain a suite of the quasi-

stationary solutions. I computed the angular momentum flux in different components of the flow to compare results in the cases with different stellar magnetic field strengths.

In a discussion of the results, I find a trend in matter density along a vertical line in the middle of the disk in the first example. The density increases with increasing stellar magnetic field in the simulation.

The second result is a trend in angular momentum flux onto the star with increasing stellar rotation rate. I find that with faster stellar rotation, the spin-up of the star decreases and eventually switches to a spin-down.

The third finding from the simulations is that for $\alpha_{\mathrm{m}}=0.1$ and a faster-rotating star, the angular momentum flux in the 

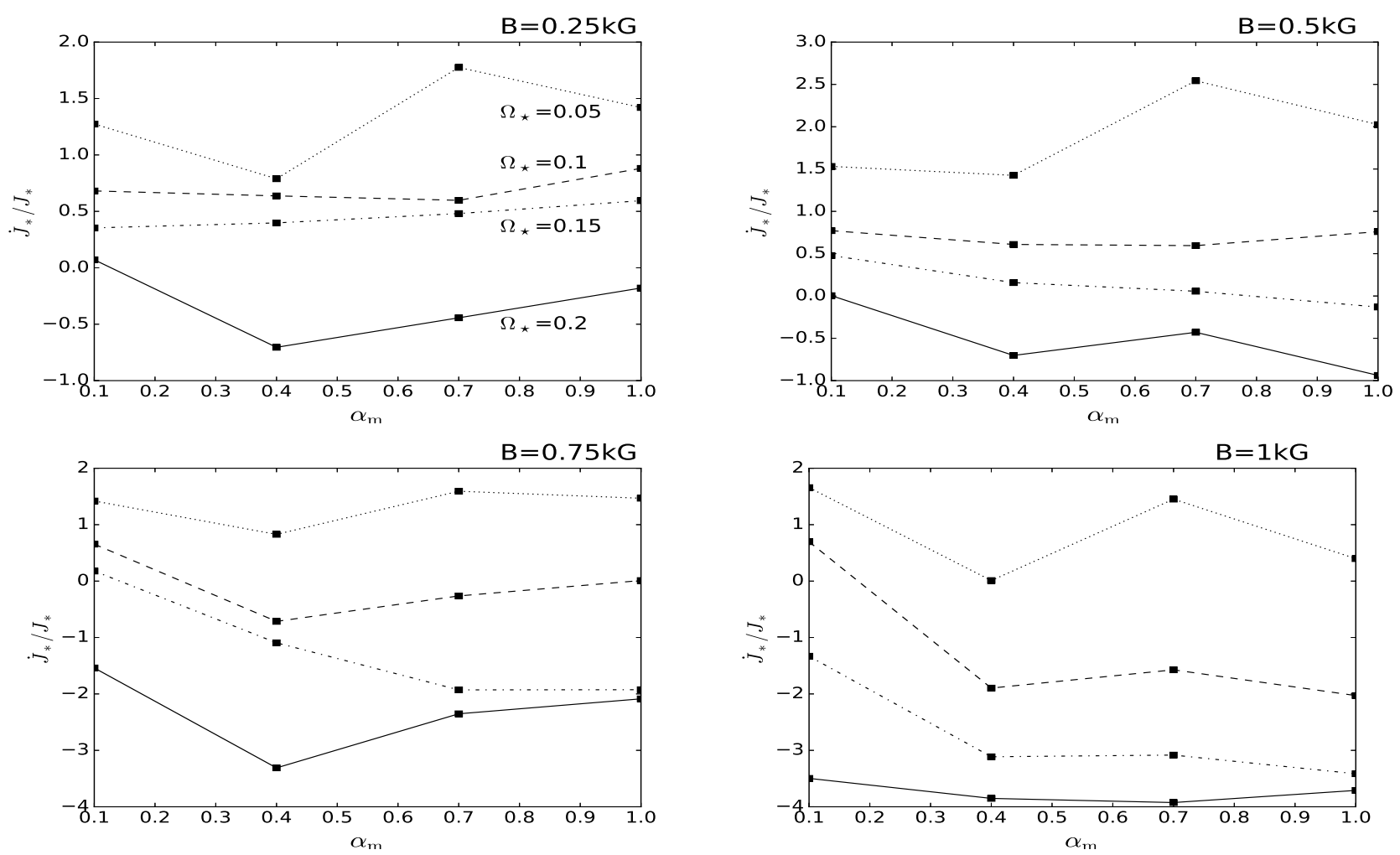

Fig. 4. Average angular momentum flux transported onto the stellar surface by the matter in-falling from the disk onto the star through the accretion column. Each panel shows a set of solutions with one stellar magnetic field strength and varying stellar rotation rate and resistivity. Results with $\Omega_{\star} / \Omega_{\mathrm{br}}=0.05$ (dotted), 0.1 (dashed), 0.15 (dash-dot-dotted), and 0.2 (solid) are shown in units of stellar angular momentum $J_{\star}=k^{2} M_{\star} R_{\star}^{2} \Omega_{\star}$ (with $k^{2}=0.2$ for the typical normalized gyration radius of a fully convective star). A positive flux spins the star up, a negative flux slows it down. With the increase in stellar rotation rate, spin-up of the star by the infalling matter decreases and eventually switches to spin-down.

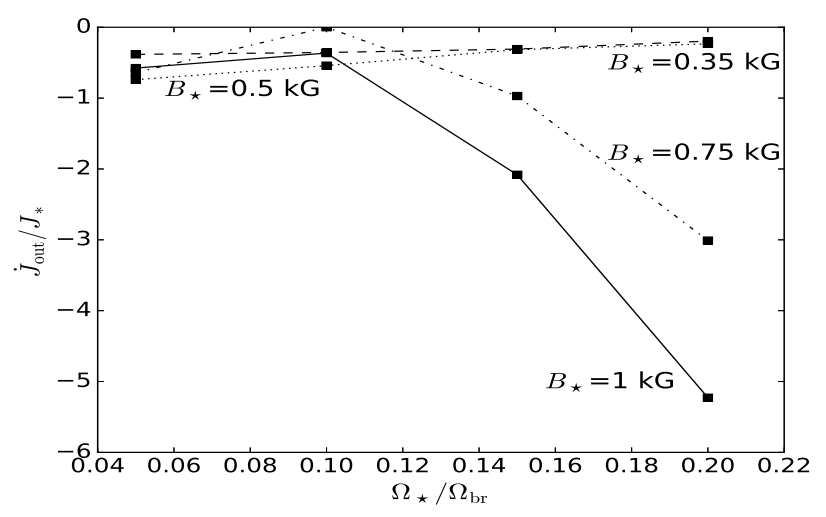

Fig. 5. Average angular momentum flux in the outflow that forms for $\alpha_{\mathrm{m}}=0.1$. It is computed at $R=12 R_{\star}$ for different stellar rotation rates. Normalization is the same as in Fig. 4. Fluxes in $B_{\star}=0.25$ (dotted), 0.5 (dash-dotted), 0.75 (dashed) and $1 \mathrm{kG}$ (solid) are shown.

conical outflow increases significantly with high values of the stellar magnetic field. The increase is about an order of magnitude. For a low stellar magnetic field, the angular momentum in the outflow remains the same.

The results apply to disks around YSOs and can be rescaled to disks in a compact binary. I provide a table with scaling factors for different cases.

I presented here the results for a slowly rotating star. In the simulations with a stellar rotation faster than $20 \%$ of the stellar breakup rotation rate, two additional solutions are obtained, similar to Romanova et al. (2009): a fast axial outflow, and with both the conical and axial outflow. I leave them for a separate study, together with the solutions with $\alpha_{\mathrm{v}}<0.685$, which show a backflow in the initial disk.

Acknowledgements. MČ developed the setup of star-disk simulations while in CEA, Saclay, France, under the ANR Toupies grant with A.S. Brun. Work in NCAC Warsaw is funded by a Polish NCN grant no. 2013/08/A/ST9/00795, and collaboration with Croatian STARDUST project through HRZZ grant IP2014-09-8656 is acknowledged. I thank IDRIS (Turing cluster) in Orsay, France, ASIAA/TIARA (PL and XL clusters) in Taipei, Taiwan and NCAC (PSK cluster) in Warsaw, Poland, for access to the Linux computer clusters I used for the high-performance computations. The PLuTo team is thanked for the possibility to use the code, in particular C. Zanni for help with the code modifications. V. Parthasarathy and F. Bartolić are acknowledged for developing the Python scripts for visualization, N. Bessolaz for the initial version of the setup, and M. Flock for useful discussions about the code.

\section{References}

Čemeljić, M., Parthasarathy, V., \& Kluźniak, W. 2017, J. Phys. Conf. Ser., 932, 012028

Ghosh, P., \& Lamb, F. K. 1979a, ApJ, 232, 259

Ghosh, P., \& Lamb, F. K. 1979b, ApJ, 234, 296

Kley, W., \& Lin, D. N. C. 1992, ApJ, 397, 600

Kluzniak, W., \& Kita, D. 2000, ArXiv e-prints [arXiv:astro-ph/0006266]

Mignone, A., Bodo, G., Massaglia, S., et al. 2007, ApJS, 170, 228

Mignone, A., Zanni, C., Tzeferacos, P., et al. 2012, ApJS, 198, 7

Powell, K. G., Roe, P. L., Linde, T. J., Gombosi, T. I., \& De Zeeuw, D. L. 1999, J. Comput. Phys., 154, 284

Prendergast, K. H., \& Burbidge, G. R. 1968, ApJ, 151, L83

Romanova, M. M., Ustyugova, G. V., Koldoba, A. V., \& Lovelace, R. V. E. 2009, MNRAS, 399, 1802

Romanova, M. M., Ustyugova, G. V., Koldoba, A. V., \& Lovelace, R. V. E. 2013, MNRAS, 430, 699

Shakura, N. I., \& Sunyaev, R. A. 1973, A\&A, 24, 337

Tanaka, T. 1994, J. Comput. Phys., 111, 381

Urpin, V. A. 1984, Sov. Astron., 28, 50

Zanni, C., \& Ferreira, J. 2009, A\&A, 508, 1117

Zanni, C., \& Ferreira, J. 2013, A\&A, 550, A99 


\section{Appendix A: Numerical simulations of star-disk magnetospheric interaction}

Star-disk magnetospheric interaction (SDMI) determines the angular momentum transfer in a star-disk system. Simulations including SDMI have been reported by Romanova et al. (2009, 2013) with a code that is not publicly available, and ZF09 and Zanni \& Ferreira (2013) with the publicly available code PLUTo (v.3). Following ZF09, I performed simulations with the updated version of the PLuTo code (v.4.1; Mignone et al. 2007, 2012). I present a short version of the setup, amended to facilitate reproducibility ${ }^{1}$.

The equations solved in the magnetohydrodynamic (MHD) module of the PLUTo code are in the cgs system of units:

$$
\begin{aligned}
& \frac{\partial \rho}{\partial t}+\nabla \cdot(\rho \boldsymbol{v})=0 \\
& \frac{\partial \rho \boldsymbol{v}}{\partial t}+\nabla \cdot\left[\rho \boldsymbol{v} \boldsymbol{v}+\left(P+\frac{\boldsymbol{B} \cdot \boldsymbol{B}}{8 \pi}\right) \boldsymbol{I}-\frac{\boldsymbol{B} \boldsymbol{B}}{4 \pi}-\boldsymbol{\tau}\right]=\rho \boldsymbol{g} \\
& \frac{\partial E}{\partial t}+\nabla \cdot\left[\left(E+P+\frac{\boldsymbol{B} \cdot \boldsymbol{B}}{8 \pi}\right) \boldsymbol{v}-\frac{(\boldsymbol{v} \cdot \boldsymbol{B}) \boldsymbol{B}}{4 \pi}\right] \\
& +\nabla \cdot[\underbrace{\eta_{\mathrm{m}} \boldsymbol{J} \times \boldsymbol{B} / 4 \pi-\boldsymbol{v} \cdot \boldsymbol{\tau}}_{\text {heating terms }}]=\rho \boldsymbol{g} \cdot \boldsymbol{v}-\underbrace{\Lambda}_{\text {cooling }} \\
& \frac{\partial \boldsymbol{B}}{\partial t}+\nabla \times\left(\boldsymbol{B} \times \boldsymbol{v}+\eta_{\mathrm{m}} \boldsymbol{J}\right)=0
\end{aligned}
$$

where the symbols have their usual meaning: $\rho$ and $\boldsymbol{v}$ are the matter density and velocity vector, $\mathrm{P}$ is the pressure, $\boldsymbol{B}$ is the magnetic field, and $\eta_{\mathrm{m}}$ and $\tau$ represent the resistivity and the viscous stress tensor, respectively. $\Lambda$ is the cooling source term, the gravity acceleration is $g=-\nabla \Phi_{\mathrm{g}}$, where the gravitational potential of the star with mass $M_{\star}$ is equal to $\Phi_{\mathrm{g}}=-G M_{\star} / R$. Then $g_{\mathrm{R}}=-1.0 / R^{2}$ in the code units.

Simulations were performed using the second-order piecewise linear reconstruction, with a Van Leer limiter in density and magnetic field and a minmod limiter in pressure and velocity. To enhance stability, in the subroutine plm_states, the default was set to Van Leer instead of the less diffusive monotonized central difference limiter. An approximate Roe solver (hlld in the PLUTO options) was used, with a modification in the flag_shock subroutine: flags were set to switch to more diffusive hll solver when the internal energy was lower than $1 \%$ of the total energy, instead of switching in the presence of shocks. The second-order timestepping (RK2) was employed, and $\nabla \cdot \boldsymbol{B}=0$ was maintained by the constrained transport. The magnetic field was evolved with the split-field option, so that only changes from the initial stellar magnetic field were evolved in time (Tanaka 1994; Powell et al. 1999). In version 4.1 of PLUTo the constrained transport is by default disabled to work with the background field in the resistive MHD. This is probably an artifact from the older version. To enable the background field option, the constraint was removed by changing the condition in the routine backgrnd_field, to point to some feature that will not be used, for instance, thermal conduction.

A logarithmically stretched radial grid and a uniform grid in the meridional half-plane $\theta=[0, \pi / 2]$ in spherical coordinates

\footnotetext{
1 I am aware of several unsuccessful attempts in repeating the ZF09 setup by experienced researchers and students in the past decade. To my knowledge, the first successful simulations following ZF09 were reported by Čemeljić et al. (2017).
}

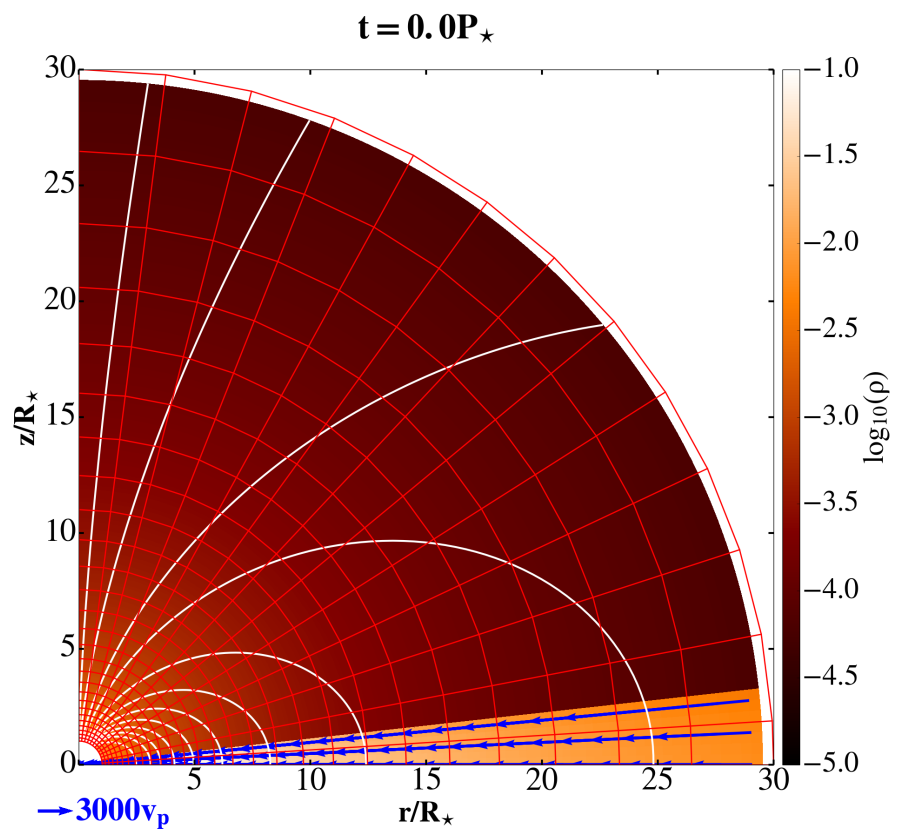

Fig. A.1. Initial density distribution of matter shown in a logarithmic color grading. A sample of the initial poloidal magnetic field lines is shown with solid white lines. Arrows show the initial velocity distribution in the disk, with the unit vector length measured in Keplerian velocity units indicated below the figure. The computational grid is shown in $8 \times 8$ blocks of cells.

were used to perform 2D axisymmetric SDMI simulations. The resolution was set to $R \times \theta=(217 \times 100)$ grid cells in the physical domain from the stellar surface to 30 stellar radii, as shown in Fig. A.1.

The total energy density is $E=P /(\gamma-1)+\rho(\boldsymbol{v} \cdot \boldsymbol{v}) / 2+\boldsymbol{B}$. $\boldsymbol{B} / 8 \pi$, where $\gamma=5 / 3$ is the plasma polytropic index. The electric current is given by Ampère's law $\boldsymbol{J}=\nabla \times \boldsymbol{B} / 4 \pi$.

To prevent the thermal thickening of the accretion disk in simulations, following the description of ZF09, I removed the underbraces Ohmic and viscous heating terms from the PLUTO energy equation. This equals the assumption that all the heating is radiated away from the disk. To do this, lines in the code with the viscous and resistive part of the flux computation in parabolic_flux subroutine were commented out. The viscous and resistive fluxes were added in the rhs subroutine to ensure that the correct dissipative flux terms were incorporated. These simulations remain in the non-ideal MHD regime because of the viscous term in the momentum equation and the resistive term in the induction equation ${ }^{2}$.

The initial disk was set with the initial density by KK00 with a self-similar profile and the aspect ratio $\epsilon$ :

$$
\begin{aligned}
\rho_{\mathrm{d}} & =\rho_{\mathrm{d} 0}\left\{\frac{\gamma-1}{\gamma \epsilon^{2}}\left[\frac{R_{*}}{R}-\left(1-\frac{\gamma \epsilon^{2}}{\gamma-1}\right) \frac{R_{\star}}{R \sin \theta}\right]\right\}^{1 /(\gamma-1)} \\
& =\rho_{\mathrm{d} 0}\left\{\frac{2}{5 \epsilon^{2}}\left[\frac{R_{\star}}{R}-\left(1-\frac{5}{2} \epsilon^{2}\right) \frac{R_{\star}}{R \sin \theta}\right]\right\}^{3 / 2} .
\end{aligned}
$$

\footnotetext{
2 Without modifying the energy equation, but with the power-law cooling introduced to account for the disk dissipative heating, a similar outcome is obtained. It is advisable to use this simpler method in the first attempt of the setup.
} 
The pressure was

$$
\begin{aligned}
& P_{\mathrm{d}}=\epsilon^{2} \rho_{\mathrm{d} 0} v_{\mathrm{K} \star}^{2}\left(\frac{\rho_{\mathrm{d}}}{\rho_{\mathrm{d} 0}}\right)^{\gamma}= \\
& =\frac{\rho_{\mathrm{d} 0} v_{\mathrm{K} \star}^{2}}{\epsilon^{3}}\left\{\frac{2}{5}\left[\frac{R_{\star}}{R}-\left(1-\frac{5 \epsilon^{2}}{2}\right) \frac{R_{\star}}{R \sin \theta}\right]\right\}^{5 / 2} .
\end{aligned}
$$

The capital $R$ indicates the spherical radius, and $r=R \sin \theta$ is the cylindrical radius. The disk unit density $\rho_{\mathrm{d} 0}$ and Keplerian speed $v_{\mathrm{K} \star}=\sqrt{G M_{\star} / R_{\star}}$ were both calculated in the disk midplane at $R_{\star}$. The initial disk was truncated about the corotation radius.

The obtained disk is a polytropic hydrodynamical solution of the viscous accretion disk in full $3 \mathrm{D}$, obtained by approximate expansion up to the second order in the terms of $\epsilon=c_{\mathrm{s}} / v_{\mathrm{K}}$, the disk aspect ratio measured on the midplane of the disk, where $c_{\mathrm{S}}=\sqrt{P_{\mathrm{d}} / \rho_{\mathrm{d}}}$ and $v_{\mathrm{K}}$ are the isothermal sound speed and Keplerian speed in the disk.

The viscosity and resistivity were defined explicitly in separate subroutines by a second-order finite-difference approximation for the dissipative fluxes, with checking of the time step. Both are parameterized by the Shakura-Sunyaev prescription as $\alpha c_{\mathrm{s}}^{2} / \Omega$. To avoid the issues related to the backflow in the disk, I here address only the case with anomalous ${ }^{3}$ viscosity coefficient $\alpha_{\mathrm{v}}=1$.

The viscosity was defined by the viscous stress tensor

$$
\boldsymbol{\tau}=\eta_{\mathrm{v}}\left[(\nabla \boldsymbol{v})+(\nabla \boldsymbol{v})^{T}-\frac{2}{3}(\nabla \cdot \boldsymbol{v}) \boldsymbol{I}\right]
$$

with the dynamic viscosity $\eta_{\mathrm{v}}=\rho v_{\mathrm{v}}$ given with

$\eta_{\mathrm{v}}=\frac{2}{3} \rho \alpha_{\mathrm{v}}\left[\left.c_{\mathrm{s}}^{2}(r)\right|_{z=0}+\frac{2}{5}\left(\frac{G M_{\star}}{R}-\frac{G M_{\star}}{r}\right)\right] \sqrt{\frac{r^{3}}{G M_{\star}}}$,

where $v_{\mathrm{v}}$ is the kinematic viscosity. The magnetic diffusivity was assumed proportional to the viscosity, with the free parameter $\alpha_{\mathrm{m}}$ :

$v_{\mathrm{m}}=\frac{3}{2} \alpha_{\mathrm{m}} \frac{v_{\mathrm{v}}}{\alpha_{\mathrm{v}}}$,

so that in the cgs system of units the resistivity is $\eta_{\mathrm{m}}=4 \pi \nu_{\mathrm{m}}$.

The diffusive parameters $\alpha_{\mathrm{v}}$ and $\alpha_{\mathrm{m}}$ were defined in separate subroutines visc_nu and res_eta. A condition for including the diffusive term was in both routines defined by the $\beta=$ $P_{\text {mag }} / P_{\text {hyd }}>0.5$, meaning that the magnetic pressure prevailed. In both subroutines, the diffusive term was taken into account only when the tracer value was unity, otherwise it was set to zero.

The initial disk velocity profile is, following KK00:

$$
\begin{aligned}
& v_{R d}=-\alpha_{\mathrm{v}} \epsilon^{2}\left[10-\frac{32}{3} \Lambda \alpha_{\mathrm{v}}^{2}-\Lambda\left(5-\frac{1}{\epsilon^{2} \tan ^{2} \theta}\right)\right] \sqrt{\frac{G M_{*}}{R \sin ^{3} \theta}}, \\
& v_{R \varphi}=\left[\sqrt{1-\frac{5 \epsilon^{2}}{2}}+\frac{2}{3} \epsilon^{2} \alpha_{\mathrm{v}}^{2} \Lambda\left(1-\frac{6}{5 \epsilon^{2} \tan ^{2} \theta}\right)\right] \sqrt{\frac{G M_{*}}{R \sin \theta}}
\end{aligned}
$$

where

$$
\Lambda=\frac{11}{5} /\left(1+\frac{64}{25} \alpha_{\mathrm{v}}^{2}\right) \text {. }
$$

\footnotetext{
3 Anomalous diffusive coefficients are much larger than coefficients computed from the microscopic considerations. They are usually given as free parameters in the simulations, motivated by the assumption that dissipation is a result of macroscopic instabilities, i.e., turbulence.
}

The initial corona is a non-rotating, polytropic corona with $\gamma=5 / 3$ in hydrostatic balance. It is defined by the density and pressure, given by

$\rho_{\mathrm{c}}=\rho_{\mathrm{c} 0}\left(R_{\star} / R\right)^{1 /(\gamma-1)}$,
$P_{\mathrm{c}}=\rho_{\mathrm{c} 0} \frac{\gamma-1}{\gamma} \frac{G M_{\star}}{R_{\star}}\left(\frac{R_{\star}}{R}\right)^{\gamma /(\gamma-1)}$,

where $\rho_{\mathrm{c} 0} \ll \rho_{\mathrm{d} 0}$ is the ratio between the initial coronal and disk density, set to 0.01 .

The initial stellar magnetic dipole field was set with the field axis aligned with the stellar rotation axis. There is no resistivity in the magnetosphere outside of the disk. This means that the reconnection of the magnetic field is a numerical, not physical dissipation.

In the internal boundary part, which enables change in the quantities inside the computational domain in PLUTO, the density in the grid cell just above the star was corrected when it falls below some limit value (I set it to $5 \times 10^{-8}$ ), to prevent the density near the star from becoming too low. The pressure was corrected in such a way to conserve the same sound speed in the corona. To maintain the self-consistency, velocities were also changed to conserve the momentum. Here was also ensured that the scalar tracer value was always set to zero in the corona. Around the reconnection sheet and outflows, the tracer scalar can obtain spurious values, this is prevented here.

The numerical heating in the corona was prevented by enforcing the conservation of the entropy $S$, to keep the values close to the initial conditions. The maximum of the entropy is on top of the star, so it was kept throughout the computational box below the value $S_{\max }=P_{\mathrm{c}} / \rho_{\mathrm{c}}^{\gamma}=2 \rho_{\mathrm{c} 0}^{-2 / 3} /\left(5 R_{*}\right)=8.61774$. For the minimum, the small number $S_{\text {min }}=0.01$ was set. From this I obtained corrected values for the pressure interval in the computational box, by $P=\max \left[\min \left(P, S_{\max } \rho^{\gamma}\right), S_{\min } \rho^{\gamma}\right]$.

In the inner boundary conditions, the density, pressure, and toroidal components of the velocity and magnetic field were prescribed from the active zones into the boundary. The linear extrapolation was used, with Van Leer limiter in the density and the magnetic field, and minmod limiter in the pressure and velocity. For the numerical stability in the corona, in the cases with $v_{\mathrm{R}}>0$, I introduced a correction of the pressure by a free parameter $T_{\mathrm{f}}$ in the inner radial boundary condition. It should be set to the number of order a few hundred. It adjusts the pressure $\rho v^{2}$ on top of the star (stellar wind) from the default $2 / 5$ to $2 / 5-T_{\mathrm{f}} v_{\mathrm{R}}^{2}$ so that the pressure in the corona is given by $P=\left(2 / 5-T_{\mathrm{f}} v_{\mathrm{R}}^{2}\right) \rho_{\mathrm{c}} R_{i}^{-5 / 2}$. The first term here is the coronal initial pressure $P_{\mathrm{c}}=2 \rho_{\mathrm{c}} R_{i}^{-5 / 2} / 5$. Because $P \sim \rho T$ in the ideal gas law, we can think of $2 / 5$ as an effective temperature, and $T_{\mathrm{f}} v_{\mathrm{R}}^{2}$ is an ad hoc correction.

In the axisymmetric 2D setup, only the toroidal component of the magnetic field was set in the staggered grid because in the constrained transport method, a staggered grid is used ${ }^{4}$. I used a custom boundary condition for $B_{\varphi}$, in which $\Omega_{\mathrm{eff}}=\Omega-v_{\mathrm{p}} B_{\varphi} / r B_{\mathrm{p}}$ is derived from the condition for the stellar surface as a rotating perfect conductor. The magnetic torque to drive the plasma rotation on top of the star was set, with the matching measured by the comparison of the stellar angular velocity and the effective rotation rate of the field lines by the ratio $\Omega_{\mathrm{eff}} / \Omega_{*}$. This prescribes

4 When the staggered grid is used, in the boundary subroutine one has to correct the misplaced call to the user-defined boundary at the grid cell centers, to come after the assignment of the normal component of staggered $\boldsymbol{B}$, instead before it. 
Table A.1. Typical values and scaling for different central objects.

\begin{tabular}{lccc}
\hline \hline & YSOs & WDs & NSs \\
\hline$M_{\star}\left(M_{\odot}\right)$ & 0.5 & 1 & 1.4 \\
$R_{\star}$ & $2 R_{\odot}$ & $5000 \mathrm{~km}$ & $10 \mathrm{~km}$ \\
$P_{\star}$ & $4.6 \mathrm{~d}$ & $6.1 \mathrm{~s}$ & $0.46 \mathrm{~ms}$ \\
$B_{\star}(\mathrm{G})$ & 500 & $5 \times 10^{5}$ & $10^{8}$ \\
\hline$\rho_{\mathrm{d} 0}\left(\mathrm{~g} \mathrm{~cm}^{-3}\right)$ & $1.2 \times 10^{-10}$ & $9.4 \times 10^{-9}$ & $4.6 \times 10^{-6}$ \\
$v_{0}\left(\mathrm{~km} \mathrm{~s}^{-1}\right)$ & 218 & 5150 & 136000 \\
$\dot{M}_{0}\left(M_{\odot} \mathrm{yr}^{-1}\right)$ & $5.7 \times 10^{-7}$ & $1.9 \times 10^{-9}$ & $10^{-9}$ \\
$B_{0}(\mathrm{G})$ & 200 & $5 \times 10^{4}$ & $2.93 \times 10^{7}$ \\
\hline
\end{tabular}

Notes. The mass $M \star$, radius $R \star$, period $P \star$ and equatorial stellar magnetic field $B_{\star}$ are chosen to derive the rest of the quantities. The code units should be multiplied by the factors given in the table to apply them to different cases.

rotation of the matter on top of the star and the effective rotation rate of the field lines to $\Omega_{\mathrm{eff}}$, with $v_{\mathrm{p}}$ and $B_{\mathrm{p}}$ standing for the poloidal velocity and magnetic field, respectively. In addition, in the constrained transport method, the subroutine ct toroidal component of the EMF was set to zero. Then in the stellar reference frame, the electric field is zero, with the flow speed parallel to the magnetic field.

I did not follow the suggestion in ZF09 to impose continuity of the speed along the field lines on the outflowing matter, nor the continuity of the axisymmetric MHD invariant $k=4 \pi \rho v_{\mathrm{p}} / B_{\mathrm{p}}$ on the infalling material, as it did not improve the result.

The stellar rotation rate was set with those corrections. I started the simulation with $\Omega_{\star}$, not with the slower rotation of the star as described in ZF09.

In the outer boundary conditions, in the coronal part of the domain, the logarithmic extrapolation in the density and pressure was set. In the radial and meridional components of the velocity an outflow was set, and a linear extrapolation with the minmod limiter for the azimuthal velocity component and Van Leer limiter in the toroidal magnetic field component. At the disk outer radial boundary, I introduced the initial hydrodynamical values, anticipating the thickening of the disk for $25 \%$. Because the velocity might roll back above the thickened disk, inflow to twice the initial disk height in the corona was prevented by
Table A.2. Position of the light cylinder as a function of stellar rotation rate in some typical cases.

\begin{tabular}{lccc}
\hline \hline$\Omega_{\star} / \Omega_{\mathrm{br}}$ & $R_{\ell \mathrm{c}}(\mathrm{YSO})$ & $R_{\ell \mathrm{c}}(\mathrm{WD})$ & $R_{\ell \mathrm{c}}(\mathrm{NS})$ \\
\hline 0.05 & 27454 & 1164 & 44 \\
0.1 & 13727 & 582 & 22 \\
0.2 & 6864 & 291 & 11 \\
0.25 & 9151 & 233 & 8.8 \\
0.5 & 2745 & 116 & 4.4 \\
0.75 & 1830 & 78 & 2.9 \\
1.0 & 1373 & 58 & 2.2 \\
\hline
\end{tabular}

Notes. Stellar rotation rate is expressed in units of stellar breakup rotation rate, and the position of the light cylinder in units of stellar radius $R_{\star}$.

setting the velocities to zero. The toroidal magnetic field was linearly extrapolated with a Van Leer limiter.

The simulations were stopped after 100 stellar rotations. In some cases, the simulation stopped earlier because the time step was too short. This was caused by the numerical instability in the rarefied corona, not by the disk instability.

Normalized equations were solved in the code. The unit length, velocity, and mass were chosen with the stellar radius $R_{\star}$, the Keplerian speed at the stellar surface $v_{\mathrm{K} \star}$, and mass $M_{\star}$. The time unit was then $t_{0}=R_{\star} / v_{\mathrm{K} \star}$. Time $t$ in the results was measured in the number of stellar rotation periods $P_{\star}$. The mass flux rate was measured in $\dot{M}_{0}=\rho_{\mathrm{d} 0} R_{\star}^{2} v_{\mathrm{K} \star}$, which was the free parameter in simulation from which I determined $\rho_{\mathrm{d} 0}$, the disk density. The initial coronal density was defined as a free parameter in the code, $\rho_{\mathrm{c} 0}=0.01 \rho_{\mathrm{d} 0}$. The magnetic field unit was defined by $B_{0}=v_{\mathrm{K} \star} \sqrt{\rho_{\mathrm{d} 0}}$. The torque in the simulations was measured in units of $\dot{J}_{0}=\rho_{\mathrm{d} 0} R_{\star}^{3} v_{\mathrm{K} \star}^{2}$.

The simulations can be rescaled to different objects using the scaling coefficients from Table A.1. In the case of compact objects, the radial extension of the domain, measured from the axis of rotation, should not reach the light cylinder, $R_{\ell \mathrm{c}} \Omega_{\star}=$ $c$, where the azimuthal velocity equals the speed of light. This limitation was not mentioned in the previous publications with SDMI, so I list distances of the light cylinder in the different cases in Table A.2. 